zeaxanthin, in facilitating thermal energy dissipation ${ }^{4}$. For example, energy dissipation (NPQ) has been shown ${ }^{5}$ to be largely

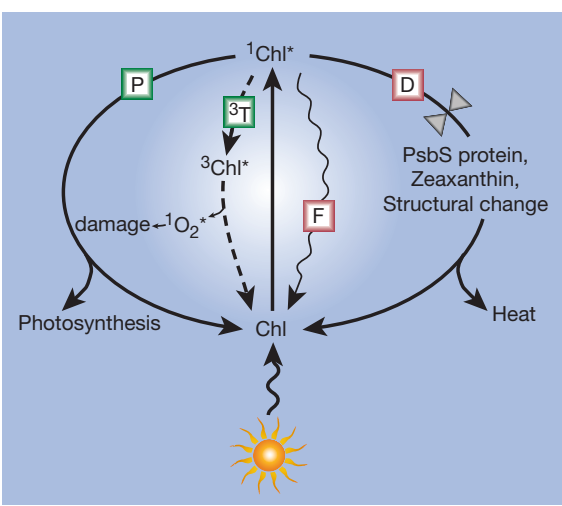

Figure 2 Fates of sunlight absorbed in the light-harvesting chlorophyll complexes. Chl, chlorophyll; ${ }^{1} \mathrm{Chl}^{\star}$, excited singlet chlorophyll; ${ }^{3} \mathrm{Chl}^{\star}$, excited triplet chlorophyll; $\mathrm{P}$, photochemistry (green); D, safe dissipation of excess excitation energy as heat (red); F, fluorescence; ${ }^{3} \mathrm{~T}$, triplet pathway, leading to the formation of singlet oxygen $\left({ }^{1} \mathrm{O}_{2}{ }^{*}\right)$ and photo-oxidative damage. absent in zeaxanthin-deficient mutants, and it is impaired in mutants that lack lutein, a close isomer of zeaxanthin. But although the zeaxanthin-deficient mutants contained increased levels of vitamin E ( $\alpha$-tocopherol, which can detoxify singlet oxygen by accepting its energy and dissipating it as heat), they showed photo-oxidative damage in the form of lipid peroxidation ${ }^{6}$. When exposed to excess light under environmental stress, leaves of these mutants developed bleached, necrotic areas and died.

This central role of zeaxanthin (and possibly lutein) in the photoprotection of photosynthesis is paralleled by a function for these same two carotenoids in photoprotection of the human eye $e^{7,8}$. In neither case, however, do we know the exact mechanism by which these carotenoids provide photoprotection. Indeed, we do not even know the location of thermal energy dissipation in the photosynthetic light-collecting system.

$\mathrm{Li}$ and colleagues' study ${ }^{1}$ is a breakthrough because it shows that, among the many different pigment-binding proteins of the photosynthetic membrane, one specific protein is essential for thermal energy dissi- pation. This protein, known as CP22 (chlorophyll-binding protein with a relative molecular mass of 22,000) or PsbS (the product of the photosystem 2 gene $p s b S$ ), had not previously been implicated in this context. The authors isolated a mutant from the higher plant Arabidopsis thaliana that contained normal levels of zeaxanthin but was deficient in energy dissipation (NPQ). Li et al. then showed that this mutant lacks the PsbS protein. They used molecular and genetic markers to pinpoint the $p s b S$ gene and reintroduced a normal copy of this gene into the mutant plant, which regained the ability to dissipate energy thermally.

Photochemistry takes place in two photosystems, and numerous different lightharvesting protein complexes funnel energy into these photosystems. Over 30 distinct members of the light-harvesting complex family have been identified ${ }^{9}$, many of which are involved in efficient light harvesting. But the function of a subgroup - which includes the PsbS protein - has not yet been worked out. Li and co-workers ${ }^{1}$ come to the important conclusion that photoprotective and light-collecting functions are not carried out

\title{
Democracy on the rocks
}

Arguably the simplest way to review the state of a subject is not to commission an article by an expert reviewer, but just to make a statistical summary of what is going on in that field. Democracy does not have the same standing as a scientific methodology as it does as a principle for government, but would an objective summary of the subjects of published papers present a very different snapshot from an expert review?

In an article entitled "The most popular rock stars, scientifically speaking" (Annals of Improbable Research $(5,20 ; 1999))$, Vidal Barrón makes a light-hearted attempt to do just this for mineralogy. Barrón analysed the titles of papers in mineralogy published from 1990 to 1996, recording the frequency of occurrence of names of minerals or mineral groups to identify the "most popular". His results are summarized in a histogram, reproduced here.

Mineralogy emerges as a subject whose remit swings rapidly from the slime to the sublime, with clays and diamond narrowly losing out to zeolites in the popularity stakes. This is an apt result because zeolites have filled both roles at different times in the eighteenth century, fine zeolite specimens were among the most valuable minerals in the known world, but their prominence today is largely practical (for ion exchange and adsorption).

All five front-runners have important industrial applications, and clearly a high proportion of mineralogical research is being carried out in industry or at its behest. Number six, perovskite, is a curious star; this is a rare oxide that few mineralogists will have handled. The key to its popularity is that its densely packed structure is believed to be typical of mineral structures at high pressures in the mantle.

Overall, the list is dominated by industrial materials, with a smattering of gemstones and intellectually

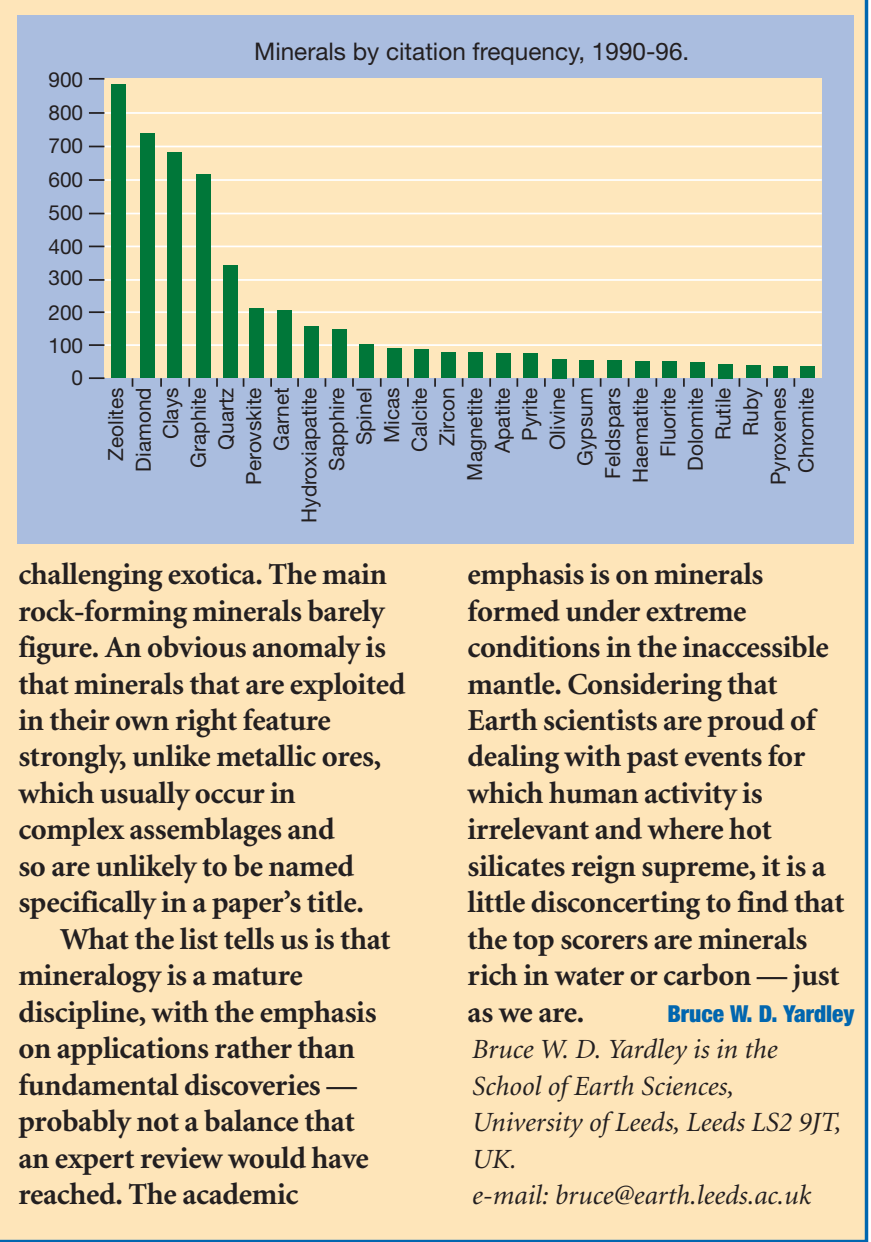

